$\begin{array}{ll} & \text { Esta obra está sob o direito de } \\ & \begin{array}{l}\text { Licença Creative Commons } \\ \text { Atribuição 4.0 Internacional. }\end{array} \\ & \end{array}$

\title{
BENEFICIOS DO PSE: A SAÚDE VAI À ESCOLA
}

Tenório Dilson Cavalcante ${ }^{1}$

José Silva de Menezes ${ }^{2}$

Betijane Soares de Barros3

\section{RESUMO}

O presente estudo objetiva-se pela explanação descritiva dos Benefícios do PSE, que apresenta um movimento de transformação entre Educação e Saúde. Metodologia: A metodologia utilizada neste trabalho será uma pesquisa descritiva, com o intuito de extrair o máximo de informações possíveis relacionadas ao tema escolhido. Resultados: Estabelecer PSE para avaliar as condições de saúde dos educandos, com ações devem considerar a visão ampliada da saúde. Conclusão: o PSE vem para permitir aos envolvidos no contexto escolar os mínimos de estrutura de saúde, através de aprendizagem, permitem a união que trará benefício a uma população muitas vezes carente de prevenção e promoção da saúde.

Palavras-chave: Promoção da Saúde. Odontologia. Serviços de Odontologia Escolar.

\footnotetext{
${ }^{1}$ Graduado em odontologia pela faculdade de odontologia de caruaru; pós-graduado em gestão de programas da saúde da família.

2 Doutorando em Ciências da Educação pela Absoulute Christian University.

${ }^{3}$ Doutora em Ciências da Educação (Faculdade de Ciências, Letras e Educação do Paraná). Doutora em Ciências da Saúde (Absoulute Christian University). Mestre em Ciências da Saúde (UFAL). Diretora do IMAS.
} 


\section{INTRODUÇÃO}

De acordo com Souza (2011) relata que a transformação em se políticas públicas na área da saúde vem com foco na atenção curativa, voltado à promoção da saúde e à melhoria da qualidade de vida, com entendimento a democratizante e universalista, integrando diferentes áreas, como a educação e a saúde.

O ministério da saúde apresenta um movimento de transformação entre Educação e Saúde, o PSE, que tem como objetivo de promoção a saúde que são estabelecidos através da educação, diante de um planejamento quanto a execução, monitoramento e a avaliação das ações realizados coletivamente, de forma a atender às necessidades e demandas locais, estabelecidos através de professores e funcionários, se faze necessário saber, o que eles sabem e o que eles podem fazer, diante do contexto estabelecido com Programa acordado, ser compostos, por, pelo menos, um representante da Secretaria de Saúde e um da Secretaria de Educação e, entretanto, pode-se haver o envolvimento de outras pessoas representantes de políticas públicas e/ou movimentos sociais (BRASIL, 2013).

Nesse processo há a necessidade de envolvimento no processo, deve-se capacitar e interpretar o cotidiano e atuar de modo a incorporar atitudes e/ou comportamentos adequados para a melhoria da qualidade de vida. Neste contexto o profissional de saúde e de educação deve ter uma postura permanente de promoção de autonomia dos princípios básicos de promoção da saúde por parte dos educandos, professores e funcionários das escolas (BRASIL, 2015).

Segundo Bordignon (2017) o PSE aborda várias temáticas, na qual suas ações devem considerar a visão ampliada da saúde, focada na sensibilização e mudança de hábitos, relacionada ao conjunto de diversos fatores como os sociais, econômicos, ambientais e políticos que determinam as condições de saúde e de qualidade de vida.

Desta forma devemos destacar a promoção a saúde, característica do PSE, que envolve as necessidades de estabelecer nos atores envolvidos no processo a capacidade de interpretar o cotidiano e atuar de modo a incorporar atitudes e/ou comportamentos adequados para a melhoria da qualidade de vida. (BRASIL, 2015).

Salum (2015) assevera que em saúde são determinantes na construção de um sistema de saúde focado para assistência, de forma educativa e gerencial. Através de ação educativa em saúde na escola é possível utilizar métodos de agir em saúde na atenção primária, em um ambiente favorável ao exercício da educação em saúde.

Segundo Bernardo (2017) o PSE permite ao seu usuário debater temas sobre 
patologias e hábitos do cotidiano como questões sexuais e a importância de uma alimentação saudável. São realizada palestras educativas, com os escolares com uma diversidade de temáticas sobre o assunto, evidenciou-se uma troca de experiências como algo extremamente satisfatório, bem como salientar que a interação estabelecida foi fundamental para criar um ambiente favorável à participação dos escolares.

Para Assad et al (2017) o processo de educativos realizado através de conversas, contempla o vínculo saúde e educação na escola. A medida que são pontuadas questões de saúde vivenciadas pelos alunos abre um lanço de compreensão entre educador e aluno na busca da melhoria da saúde dos educandos de uma comunidade, utilizando uma interlocução entre o serviço de saúde, a educação e o ambiente familiar, exerce influência ao promove ações de orientações buscamos proporcionar uma melhor qualidade.

Para Santiago (2012) o desenvolvimento do PSE depende do envolvimento de

\section{METODOLOGIA}

O presente estudo objetiva-se pela explanação de conteúdo, acerca do tema escolhido, na qual se utilizou de uma pesquisa relatando a importância do PSE (Programa Saúde na Escola), com a intenção de incluir,
Secretarias Municipais de Saúde e de Educação, que deve realizar esclarecendo o propósito do Programa, sobre sua operacionalidade mostrando a importância da parceria entre escola, professores, coordenadores e a ESF, que começam a ser consideradas como um ponto de referência para necessidades básicas, despertando-os para a preocupação com sua saúde e o auto cuidado, para que assim ocorra uma parceria e consequentemente o sucesso do programa.

Em seu relato Vieira (2014) que as ações realizadas no PSE colaboram na Estratégia de Saúde da Família (ESF), seus profissionais também participam desta condução do programa, onde é possível identificar e reconheceram a importância de ações de saúde na escola e as dificuldades na realização das atividades no ambiente escolar.

Segundo Souza (2011) o conhecimento das ações das equipes da Estratégia de Saúde da Família (ESF) e que as equipes pelos professores são primordiais na consolidação do PSE, entender as propostas da escola para que possa existir um diálogo entre os dois setores.

tanto com experiências, como novos conhecimentos, que servirão como base e apoio ao decorrer do trabalho. 
A metodologia utilizada neste trabalho será uma pesquisa sistemática, com o intuito de extrair o máximo de informações possíveis relacionadas ao tema escolhido.

Uma pesquisa descritiva colabora de forma a registrar, analisar e identificar dados que se referem ao assunto, podendo ser utilizadas de várias técnicas de pesquisa, como internet, livros e revistas.

Contudo, o trabalho será desenvolvido de forma a descrever os impactos e a importância que a Programa Saúde na Escola, analisando a conceituação teórica, com diversas linhas de pensamentos e relatando a teoria para a realidade diária no processo de ações de saúde aos alunos da rede pública de ensino.
Para esta revisão foram utilizados como meios de consultas, artigos científicos da base de dados virtuais: PubMed (Public Medline), MEDLINE (Sistema Online de Busca e Análise de Literatura Médica), SciElo (Scientific Eletronic Library Online), LILACS (Literatura Latino-Americana e do Caribe em Ciências da Saúde), via portal BVS (Biblioteca Virtual em Saúde), bem como revistas científicas e consultas complementares ao acervo da biblioteca central do Centro Universitário Tiradentes (UNITAL). Na Tabela 1 encontram-se os detalhes numéricos das pesquisas realizadas nas diversas bibliotecas consultadas.

Tabela 1: Características dos estudos selecionados sobre o PSE (Programa Saúde na Escola).

\begin{tabular}{l|l|l|l}
\hline BASE DE DADOS & TOTAL DE REFERENCIAS & ARTIGOS EXCLUIDOS & $\begin{array}{l}\text { ARTIGOS } \\
\text { SELECIONA- } \\
\text { DOS }\end{array}$ \\
\hline LILACS & 14 & 12 & 3 \\
\hline SCIELO & 65 & 62 & 3 \\
\hline MEDLINE & 370 & 368 & 3 \\
\hline PUBMED & 129 & 127 & 3 \\
\hline BVS & & & 2 \\
\hline
\end{tabular}

Fonte: adaptado de Fregadolli (2020). 


\section{Detalhamento das etapas da revisão sistemática integrativa}

Quadro 1: Etapas da Revisão Sistemática.

\begin{tabular}{|c|c|c|c|}
\hline $\begin{array}{l}\text { TÓPICOS DE CADA } \\
\text { ETAPA }\end{array}$ & \multicolumn{3}{|c|}{ DETALHAMENTO DE CADA TÓPICO } \\
\hline Tema & \multicolumn{3}{|c|}{ Beneficios do pse: a saúde vai à escola } \\
\hline Pergunta norteadora & \multicolumn{3}{|c|}{ Quais os benefícios do PSE? } \\
\hline Objetivo geral & \multicolumn{3}{|c|}{$\begin{array}{l}\text { Discutir através de levantamentos bibliográficos os beneficios da PSE no } \\
\text { universo escolar. }\end{array}$} \\
\hline Estratégias de busca & $\begin{array}{l}\text { 1. Cruzamento } \\
\text { NOT, OR; } \\
\text { 2. Uso de aspas } \\
\text { para que a varredura } \\
\text { Quadro; } \\
\text { 3. } \quad \text { Uso de descr } \\
\text { 4. } \quad \text { Uso de meta } \\
\text { 5. } \quad \text { 5. Uso de de }\end{array}$ & $\begin{array}{l}\text { critores por meio do } \\
\text { olitermos (descritor } \\
\text { igos científicos con } \\
\text { estruturados (codifi } \\
\text { (filtros) nas bibliote } \\
\text { es em inglês para an }\end{array}$ & $\begin{array}{l}\text { dor booleano AND, } \\
\text { ais de um termo) } \\
\text { se o termo exato- } \\
\text { no DECS ou MESH; } \\
\text { uais; } \\
\text { número de artigos. }\end{array}$ \\
\hline \multirow{3}{*}{$\begin{array}{l}\text { Bancos de terminolo- } \\
\text { gias }\end{array}$} & Banco & \multicolumn{2}{|c|}{ Link } \\
\hline & $\mathbf{C}$ & \multicolumn{2}{|c|}{ http://decs.bvs.br/ } \\
\hline & $\mathrm{H}$ & \multicolumn{2}{|c|}{ https://www.ncbi.nlm.nih.gov/mesh } \\
\hline \multirow[t]{4}{*}{$\begin{array}{l}\text { Descritores livres e } \\
\text { estruturados }\end{array}$} & Descritor & DeCS (Registro) & $\begin{array}{l}\text { MeSH (Identifica- } \\
\text { dor Único) }\end{array}$ \\
\hline & Promoção da Saúde & 6444 & D006293 \\
\hline & Odontologia & 3812 & D003797 \\
\hline & $\begin{array}{l}\text { Serviços de Odon- } \\
\text { tologia Escolar }\end{array}$ & 12952 & $\mathrm{D} 012570$ \\
\hline String de busca & \multicolumn{3}{|c|}{ Educação AND Saúde AND Escola } \\
\hline \multirow[t]{2}{*}{ Bibliotecas Virtuais } & Biblioteca & \multicolumn{2}{|c|}{ Link } \\
\hline & $\begin{array}{c}\text { BVS } \\
\text { LILACS } \\
\text { SCIELO } \\
\text { MEDLINE } \\
\text { PUBMED }\end{array}$ & \multicolumn{2}{|c|}{$\begin{array}{c}\text { https://bvsalud.org/ } \\
\text { https://lilacs.bvsalud.org/en/ } \\
\text { https://scielo.org/ } \\
\text { http://bases.bireme.br/ }\end{array}$} \\
\hline
\end{tabular}

Fonte: adaptado de Fregadolli (2020).

Quadro 2: síntese de busca.

\begin{tabular}{|l|c|c|l|}
\hline \multicolumn{1}{|c|}{ TítULO Do ARTIGO } & $\begin{array}{c}\text { ANO DE } \\
\text { PUBLICAÇÃo }\end{array}$ & AUTOR & \multicolumn{1}{c|}{ MÉTODO APLICADO } \\
\hline $\begin{array}{l}\text { Saúde em roda: a experiência in- } \\
\text { tersetorial entre saúde e educação }\end{array}$ & 2016 & $\begin{array}{c}\text { ASSAD, } \\
\text { S.G.B. et al al }\end{array}$ & $\begin{array}{l}\text { Estudo de natureza descritiva e ex- } \\
\text { ploratória com abordagem qualita- } \\
\text { tiva }\end{array}$ \\
\hline $\begin{array}{l}\text { Caderno do gestor do PSE / Mi- } \\
\text { nistério da Saúde }\end{array}$ & 2015 & BRASIL & $\begin{array}{l}\text { Estudo de caso múltiplo transversal } \\
\text { e de cunho exploratório }\end{array}$ \\
\hline
\end{tabular}




\begin{tabular}{|c|c|c|c|}
\hline $\begin{array}{l}\text { Physical activity and nutrition ed- } \\
\text { ucation at the school environment } \\
\text { aimed at preventing childhood } \\
\text { obesity: evidence from systematic } \\
\text { reviews. }\end{array}$ & 2016 & $\begin{array}{l}\text { GUERRA, } \\
\text { P.H }\end{array}$ & $\begin{array}{l}\text { Estudo de caso múltiplo transversal } \\
\text { e de cunho exploratório. }\end{array}$ \\
\hline $\begin{array}{l}\text { Oficina sobre sexualidade na ado- } \\
\text { lescência: uma experiência da } \\
\text { equipe saúde da família com ado- } \\
\text { lescentes do ensino médio. }\end{array}$ & 2011 & $\begin{array}{l}\text { MARTINS, } \\
\text { C.B.G. et al }\end{array}$ & Estudo descritivo e exploratório. \\
\hline $\begin{array}{l}\text { Rede de atenção às urgências e } \\
\text { emergências: pré-avaliação das } \\
\text { Unidades de Pronto Atendimento } \\
\text { (UPAs) em uma região metropoli- } \\
\text { tana do Brasil. }\end{array}$ & 2012 & SILVA GS & $\begin{array}{l}\text { Estudo quantitativo, descritivo e } \\
\text { transversal. }\end{array}$ \\
\hline Educação e saúde na escola. & 2011 & SOUZA AA, & Estudo descritivo e exploratório. \\
\hline $\begin{array}{l}\text { Implantação do Programa Saúde } \\
\text { na Escola em Fortaleza - CE: atu- } \\
\text { ação de equipe da Estratégia Sa- } \\
\text { úde da Família. }\end{array}$ & 2012 & $\begin{array}{l}\text { SANTIAGO } \\
\text { LM }\end{array}$ & Estudo descritivo e exploratório. \\
\hline $\begin{array}{l}\text { Causas da não utilização de pre- } \\
\text { servativos nas práticas sexuais de } \\
\text { adolescentes: revisão integrativa. }\end{array}$ & 2016 & $\begin{array}{l}\text { BOR- } \\
\text { DIGNON, } \\
\text { M.N.F.D.; }\end{array}$ & Estudo descritivo e exploratório. \\
\hline $\begin{array}{l}\text { Atuação dos enfermeiros de uni- } \\
\text { dades básicas de saúde direcio- } \\
\text { nada aos adolescentes com ex- } \\
\text { cesso de peso nas escolas. }\end{array}$ & 2014 & $\begin{array}{l}\text { VIEIRA, } \\
\text { C.E.N.K. et } \\
\text { al }\end{array}$ & $\begin{array}{l}\text { Estudo quantitativo, descritivo e } \\
\text { transversal. }\end{array}$ \\
\hline $\begin{array}{l}\text { Ministério da Educação. Orienta- } \\
\text { ções sobre o Programa Saúde na } \\
\text { Escola para a elaboração dos pro- } \\
\text { jetos locais. }\end{array}$ & 2007 & BRASIL & $\begin{array}{l}\text { Estudo de caso múltiplo transversal } \\
\text { e de cunho exploratório. }\end{array}$ \\
\hline $\begin{array}{l}\text { Educação em saúde para adoles- } \\
\text { centes na escola: um relato de ex- } \\
\text { periência }\end{array}$ & 2015 & $\begin{array}{l}\text { SALUM, } \\
\text { G.B.; MON- } \\
\text { TEIRO, } \\
\text { L.A.S } \\
\end{array}$ & $\begin{array}{l}\text { Estudo de caso múltiplo transversal } \\
\text { e de cunho exploratório. }\end{array}$ \\
\hline $\begin{array}{l}\text { Saúde na escola: contribuições fe- } \\
\text { nomenológicas a partir da percep- } \\
\text { ção do aluno adolescente. }\end{array}$ & 2016 & $\begin{array}{l}\text { FAIAL, } \\
\text { L.C.M. et al }\end{array}$ & $\begin{array}{l}\text { Estudo quantitativo, descritivo e } \\
\text { transversal. }\end{array}$ \\
\hline $\begin{array}{l}\text { Programa Saúde nas Escolas: O } \\
\text { olhar dos Profissionais da Saúde }\end{array}$ & 2013 & $\begin{array}{l}\text { BEZERRA, } \\
\text { Italla Maria } \\
\text { Pinheiro }\end{array}$ & Estudo descritivo e exploratório \\
\hline $\begin{array}{l}\text { Projeto de Lei - Aprova o Plano } \\
\text { Nacional de Educação para o de- } \\
\text { cênio 2011- 2020, e dá outras pro- } \\
\text { vidências }\end{array}$ & 2011 & BRASIL & Estudo descritivo e exploratório \\
\hline Programa Saúde na Escola. & 2017 & BRASIL & Estudo descritivo e exploratório \\
\hline $\begin{array}{l}\text { Proposta de aplicação do diagnos- } \\
\text { tico interdisciplinar no transopera- } \\
\text { tório. }\end{array}$ & 2014 & COSTA & $\begin{array}{c}\text { Estudo quantitativo, descritivo e } \\
\text { transversal }\end{array}$ \\
\hline
\end{tabular}

Fonte: adaptado de Fregadolli (2020).

\section{As diretrizes do programa saúde na es- cola (pse)}


Segundo o ministério da saúde, Brasil (2015), as diretrizes do programa são baseadas em:

I - Tratar a saúde e educação integrais como parte de uma formação ampla para a cidadania e o usufruto pleno dos direitos humanos;

II - Permitir a progressiva ampliação Inter setorial das ações executadas pelos sistemas de saúde e de educação com vistas à atenção integral à saúde de crianças e adolescentes; III - Promover a articulação de saberes, a participação dos educandos, pais, comunidade escolar e sociedade em geral na construção e controle social das políticas públicas da saúde e educação;

IV - Promover a saúde e a cultura da paz, favorecendo a prevenção de agravos à saúde, bem como fortalecer a relação entre as redes públicas de saúde e de educação; V - Articular as ações do Sistema Único de Saúde (SUS) às ações das redes de educação pública de forma a ampliar o alcance e o impacto de suas ações relativas aos educandos e suas famílias, otimizando a utilização dos espaços, equipamentos e recursos disponíveis;

VI - Fortalecer o enfrentamento das vulnerabilidades, no campo da saúde, que possam comprometer o pleno desenvolvimento escolar;

VII - Promover a comunicação, encaminhamento e resolutividade entre escolas e unidades de saúde, assegurando as ações de atenção e cuidado sobre as condições de saúde dos estudantes;

VIII- Atuar, efetivamente, na reorientação dos serviços de saúde para além de suas responsabilidades técnicas no atendimento clínico, para oferecer uma atenção básica e integral aos educandos e à comunidade. 


\section{RESULTADOS E DISCUSSÕES}

Para Bordignon (2017) é necessário o entendimento de todos os atores envolvidos no programa, o PGE deve ser utilizado de forma estratégica visando estabelecer parcerias com os professores e capacitá-los. Dessa forma os profissionais podem promover ações de orientações, a experiência da escuta do discente subsidia uma reflexão crítica sobre a saúde na escola, auxiliado o usuário a reorientação nos moldes da assistência humanizada e holística, com amparo da educação em saúde (FAIAL, 2017).

Segue abaixo, as categorias temáticas elaboradas a partir da revisão sistemática.

\section{Aproximação entre a saúde e a educação}

Para Casemiro (2012) sempre existiu uma aproximação entre a saúde e a educação, pois ter aceso ao conhecimento permite que a população uma vida mais saudável. O ambiente escolar possibilita de iniciativas para melhor compreensão sobre temas importantes como: violência, adolescência e métodos contraceptivos, higiene pessoal, alimentação, dentre outros

Segundo o ministério da saúde o Programa Saúde na Escola (PSE) tem como objetivo ampliar as ações de saúde aos alunos da rede pública de ensino, com o objetivo da formação integral dos estudantes da
Educação Básica por meio de ações de prevenção, promoção e atenção à saúde (BRASIL, 2007). Nesse contexto o projeto é voltado escovação dental supervisionada dentro da ação coletiva, iniciativa de grande importância no debate da saúde bucal da população, que promove medidas supervisionada direcionada a grupos determinados com orientação e supervisão de um profissional treinado. (BRASIL, 2007).

No texto referente ao PSE, o ministério da saúde relata que as equipes de Saúde da Família realizarão visitas periódicas e permanentes às escolas participantes do PSE para avaliar as condições de saúde dos educandos, bem como para proporcionar o atendimento à saúde ao longo do ano letivo, de acordo com as necessidades locais de saúde identificadas (Parágrafo único, Art. $4^{\circ}$ ) “(BRASIL, 2007b). Desta forma Educação Básica e as equipes de Saúde da Família, de forma articulada entre as políticas e ações de educação e de saúde, com a participação da comunidade escolar.

O PSE em seu processo de adesão é caracterizado pelas de responsabilidades e compromissos a serem firmados entre o Ministério da Educação (MEC) e os Secretários de Saúde e Educação de cada município. Desta forma, os munícipios podem aderir ao PSE, através do preenchimento de dados no Portal do Gestor do Ministério da Saúde, em um processo que ocorre a cada dois 
anos. As responsabilidades dos setores envolvidos são estabelecidas através da adesão de um termo de compromisso (BRASIL, 2017 $)$

Guerra (2016) relata que o programa PSE, são realizadas palestras com os escolares com uma diversidade de temáticas que aborda questões sexuais, esclarecimento sobre a importância de uma alimentação saudável, troca de experiências que permite a interação, fundamental para criar um ambiente favorável à participação dos escolares. Uma das patologias abordadas é a obesidade, que apresenta riscos como as doenças cardiovasculares, problemas na artéria coronária e o acidente vascular cerebral isquêmico. A conscientização da importância das avaliações antropométricas é trabalhada dentro desse projeto, ações para um maior controle da obesidade, através das intervenções que podem ser realizadas diante da realização do Índice de Massa Corpórea (IMC).

Nesse contexto, as ações de promoção, prevenção e atenção à saúde a nível primário tem como objetivo passar informações aos estudantes que enfrentam vulnerabilidades sociais que comprometem o pleno desenvolvimento de crianças e jovens da rede pública de ensino. (BRASIL, 2013).

Perante o vínculo saúde/educação foi instituída uma nova Portaria no mês de abril do ano de 2017, que vem a definir critérios de adesão dos municípios no PSE.O presente documento tem como diretriz garantir aos estudantes a atualização do calendário vacinal e ações de promoção à saúde, bem como da prevenção à obesidade, cuidados com a saúde bucal, auditiva e ocular, combate ao mosquito transmissor da dengue, além da proposta de promover também maior incentivo à prática de atividades físicas e prevenção de DSTs (BRASIL, 2017b).

O ministério da saúde assevera sobra a descentralização e respeito à autonomia federativa são diretrizes do Programa Saúde na Escola (PSE), a integração e articulação das redes públicas de ensino e de saúde; territorialidade; interdisciplinaridade e integralidade; cuidado ao longo do tempo; controle social; monitoramento e avaliação permanentes (BRASIL, 2015).

\section{Ferramentas utilizadas nas ações do PSE}

Para Silva (2012) a integração da Educação e da Saúde no contexto escolar é de grande importância, pois desta forma integrada com dois sistemas irão promover ações preventivas voltada para a comunidade escolar, integrando os profissionais, transformando-os em grandes aliados para mostrar de forma mais adequada as questões que envolvem a sexualidade e temas afins. 
Costa (2014) relata que a promoção da saúde no ambiente escolar permite uma visão integral e multidisciplinar do indivíduo, levando em consideração todo seu entorno familiar, comunitário, social e ambiental. A realização de rodas de conversa é uma das ferramentas utilizadas nas ações do PSE, desta forma são citadas questões de saúde vivenciadas pelos alunos, medidas para melhoria da saúde dos educandos de uma comunidade, utilizando uma interlocução entre o serviço de saúde, a educação e o ambiente familiar, uma vez que este exerce influência no desempenho destas crianças e relaciona-se às dificuldades no aprendizado delas. O objetivo principal é de orientar e entender com as famílias os problemas vivenciados, na busca de uma melhor qualidade de vida para essas crianças, através de ações conjuntas entre a ESF e a Educação (ASSAD,2016).

As ações realizadas pelo PSE são estruturadas em três pilares: à avaliação das condições de saúde: à promoção de saúde e prevenção de agravos; à formação e ou capacitação de profisssionais. Neste contexto o desenvolvimento destas ações são compreendidas em essenciais e optativas, desta forma podemos entender como essenciais: avaliação antropométrica; verificação da situação vacinal; saúde bucal; acuidade visual; segurança alimentar e promoção de alimentação saudável; promoção de cultura de paz; educação para saúde sexual, saúde reprodutiva e prevenção de DST/Aids; prevenção ao uso de álcool, tabaco e outras drogas, em relação a optativa: saúde auditiva, desenvolvimento de linguagem, doenças negligenciadas, saúde mental, prevenção de acidentes, saúde ambiental, práticas corporais e atividades físicas. (BRASIL, 2015).

Para Bressan e Medeiros (2014) as ações de educação em saúde são componente de formação para a cidadania, através de estratégias de promoção da saúde com o objetivo de construção do ambiente escolar favorável à escolha pelo estilo de vida saudável.

Nesse contexto, as ações realizadas fortalecem a participação social a partir do entendimento das práticas realizadas e sua partilha, construindo saberes e práticas, autonomia e formas de participação. Desta forma a preparação das equipes de PSE e ESF deve ser primordial, pois por meio do conhecimento que pode transformar os comportamentos dos indivíduos.

Os desafios encontrados são muitos, desde a integração com ensino de competência a instrumentalização técnica dos professores e funcionários das escolas e dos profissionais da Estratégia de Saúde da Família para apoiar e fortalecer as iniciativas, outro ponto a ser abordado é vigilância de práticas de risco que visa a identificação 
com a intenção de monitorar as iniciativas, para melhorar o compromisso das escolas com a promoção da saúde de seus alunos, professores e outros membros da comunidade escolar (BRASIL, 2007a).

Segundo o ministério da saúde, o programa tem a capacidade de desenvolver integralmente estudantes e promover o acesso da comunidade escolar em programas de saúde de modo a formar cidadãos críticos e capacitados para o enfrentamento das vulnerabilidades que possam comprometer o desenvolvimento dos educandos (BRASIL, 2011).

Ferreira et al. (2014), destaca que as equipe de saúde da ESF enfrentam dificuldade pela excessiva demanda de trabalho na unidade de saúde, além das barreiras apontadas pelos profissionais em se trabalhar intersetorialmente. Desta forma diminui o vínculo estabelecido prejudicando as atividades do programa.

Para Cunha Filho e Ferreira-Borges (2008) existem quatro pilares fundamentais a serem adotados em qualquer programa de saúde, a definição clara das políticas no contexto escolar; a criação de um ambiente escolar promotor de saúde e preventivo de problemas; as atividades educativas e de desenvolvimento de habilidades. Criar condições torna-se importante no qual promova a saúde; e o desenvolvimento de serviços específicos de saúde e apoio psicossocial.
Faial (2017) entende que o programa deve evitar atendimentos isolados e esporádicos, permeados por atitudes e práticas desumanizadas com prejuízos na garantia do pleno cuidado, dessa maneira extinguir as relações que podem gerar algum conflito no ambiente escolar através de atendimentos desarticulados.

\section{O desenvolvimento de ações de promo- ção à saúde}

O ambiente escolar traz a possibilidade ao desenvolvimento de atividades que possam abordar todos os aspectos do indivíduo, sendo, portanto, local privilegiado para o desenvolvimento de ações de promoção à saúde e prevenção de doenças. O processo de aprendizagem permite a formação de cidadãos que atuam frente a direitos e deveres no contexto político, civil e social. Em um processo de conhecimento o indivíduo é conduzido a adotar hábitos de contribuição positiva à saúde, agindo de forma comprometida com a mesma no âmbito individual e coletivo, desta forma utilizar a educação em saúde para ações pontuais ou de aplicação momentânea, contribui para formação do aluno, trazendo práticas que possam ser inseridas em seu cotidiano (BRASIL, 2011).

Para Salum (2015) as práticas educativas em saúde colaboram para um sistema de saúde mais integral, articulado, fo- 
cado no assistencialismo, educativo e gerencial, as medidas educativas na saúde na escola é propicio ao exercício da educação em saúde, voltada a atenção primária em saúde.

Segundo Martins et al., (2013) a troca de informação, saúde e educação, deve ser potencializado para a consolidação de parcerias efetivas entre esses setores, juntamente com a família, desta forma a Estratégia de Saúde da Família deve focar na inclusão dos escolares nas ações da equipe e que os profissionais de saúde, afim de desenvolver uma rede e construir estratégias de intervenção de forma articulada com outros setores.

Martins (2011) alega que a interação profissionais de saúde e os educandos com a comunidade escolar ainda é limitada, desta forma PSE tem como plataforma de gestão estabelecer e manter um vínculo pautado no envolvimento dos envolvidos estabelecendo confiança entre o programa e os alunos e familiares. $\mathrm{O}$ envolvimento dos profissionais de saúde no universo escolar permite a percepção do seu papel social de educador, aproximando escola e unidade de saúde, ajudando os adolescentes e transformando a informação científica em comportamentos saudáveis.

\section{CONCLUSÃO}

O desenvolvimento desta pesquisa possibilitou conhecer a importância do a importância da implantação do PSE no universo escolar. O programa trabalha o cenário de vulnerabilidade que os escolares estão inseridos, o Ministério da Educação entende que união trará benefício a uma população muitas vezes carente de prevenção e promoção da saúde.

Obter conhecimento sobre o desenvolvimento do programa se torna necessário, entender os procedimentos aplicados a mesmo sobre uma de educador, são pontos fundamentais neste contexto, já que os desafios encontrados especialmente no que se refere ao envolvimento da comunidade escolar com a saúde básica.

É notório que o PSE vem para permitir aos envolvidos no contexto escolar os mínimos de estrutura de saúde, através de aprendizagem, tornam-se multiplicadores de informações e de opiniões, ofertando assim benefícios para uma comunidade inteira. 


\section{REFERÊNCIAS}

ASSAD, S.G.B. Saúde em roda: a experiência intersetorial entre saúde e educação. Revista de Enfermagem, v. 11, n. 1, p. 470473, 2016. ISSN: 1981-8963.

MARTINS, A. A. A.; ALBUQUERQUE, G. A.; BEZERRA, I. M. P.; ANTÃO, J. Y. F. L.; ABREU, L. C.; MACHADO, M. F. A. S.; DANTAS, M. N. L. PROGRAMA SAÚDE NAS ESCOLAS: O OLHAR DOS PROFISSIONAIS DA SAÚDE. In:

II Congresso Online de Gestão, Educação e Promoção da Saúde- II CONVIBRA SAÚDE, 2013. PROGRAMA SAÚDE NAS ESCOLAS: O OLHAR DOS PROFISSIONAIS DA SAÚDE, 2013.

BORDIGNON, M.N.F.D.; LIBERALI, R.; BORDIGNON, J.C.P. Causas da não utilização de preservativos nas práticas sexuais de adolescentes: revisão integrativa. $R e$ vista de Enfermagem, v. 11, n. 1, p. 207213, 2016. ISSN: 1981-8963.

BRASIL. Ministério da Saúde. Caderno do gestor do PSE / Ministério da Saúde, Ministério da Educação. - Brasília: Ministério da Saúde, 2015.

\section{MINISTÉRIO DA EDU-}

CAÇÃO. Manual Instrutivo. Programa Saúde na Escola. Brasília, 2013.

. Projeto de Lei - Aprova

o Plano Nacional de Educação para o decênio 2011-2020, e dá outras providências. 2011.

. Ministério da Saúde. Ministério da Educação. Orientações sobre o Programa Saúde na Escola para a elaboração dos projetos locais. Brasil, 2007. , Ministério da Saúde. Programa Saúde na Escola. Brasília, 2017a BRESSAN, A.; MEDEIROS, D. C. A. Promoção da Saúde na Escola. Revista Pátio. Rio Grande do Sul, 69 eds. 2014.
CASEMIRO, J. P.; FONSECA, A. B. C.; SECCO, F. V. M. Promover saúde na escola: reflexões a partir de uma revisão sobre saúde escolar na América Latina. Revistas Cientificas de América Latina y el Caribe, España y Portugal. Rio de Janeiro, v. 19, n. 3, p. 829-840, 2012.

COSTA, C. N. B. Proposta de aplicação do diagnostico interdisciplinar no transoperatório. Liph Science, v. 1, n. 1, jul./set., 2014

FAIAL, L.C.M. Saúde na escola: contribuições fenomenológicas a partir da percepção do aluno adolescente. Revista de enfermagem, v. 11, n. 1, p. 24-30, 2016. ISSN: 1981-8963.

GUERRA, P. H..; SILVEIRA, J. A. C.; SALVADOR, E. P. Physical activity and nutrition education at the school environment aimed at preventing childhood obesity: evidence from systematic reviews. Jornal de Pediatria (Versão em Português), v. 92, n. 1, p. 15-23, 2016.

MARTINS, C. B. G. Oficina sobre sexualidade na adolescência: uma experiência da equipe saúde da família com adolescentes do ensino médio. Revista Mineira de Enfermagem, v. 15, n. 4, p. 573-578, 2011.

Silva, G. S.; Samico, I.; Dubeux, L.S.; Felisberto $\mathrm{E}$. Redes de atenção às urgências $\mathrm{e}$ emergências: pré-avaliação das Unidades de Pronto Atendimento (UPAs) em uma região metropolitana do Brasil. Revista Brasileira de Saúde Materno Infantil. V. 12, n. 4, p. 445-458. 2012.

SOUZA, A. A.; SOUZA V.; Guerra, M. Educação e Saúde na Escola. Belo Horizonte: CAED-UFMG. 293p. il. ISBN:97885-64724-06-8. 2011.

SALUM, G. B.; MONTEIRO, L. A. S. Educação em saúde para adolescentes na escola: um relato de experiência. Revista 
Mineira de Enfermagem, v. 19, n. 2, p. 246-257, 2015.

SANTIAGO L. M.; Rodrigues, M. T. P.; Oliveira Junior, A. D.; Moreira, T. M. M. Implantação do Programa Saúde na Escola em Fortaleza - CE: atuação de equipe da
Estratégia Saúde da Família. Revista Mineira de Enfermagem, v. 65, n. 6, p. $1026-$ 1029. 2012.

VIEIRA, C.E.N.K. et al. Atuação dos enfermeiros de unidades básicas de saúde direcionada aos adolescentes com excesso de peso nas escolas. Revista Mineira de Enfermagem, v. 18, n. 3, p. 630-643, 2014. 